\title{
Concordant genotype of upper and lower airways $P$ aeruginosa and $S$ aureus isolates in cystic fibrosis
}

\author{
J G Mainz, ${ }^{1}$ L Naehrlich, ${ }^{2}$ M Schien, ${ }^{1}$ M Käding, ${ }^{3}$ I Schiller, ${ }^{1}$ S Mayr, ${ }^{4}$ G Schneider, ${ }^{5}$ \\ B Wiedemann, ${ }^{6}$ L Wiehlmann, ${ }^{7} \mathrm{~N}$ Cramer, ${ }^{7}$ W Pfister, ${ }^{8}$ B C Kahl, ${ }^{9} \mathrm{~J}$ F Beck, ${ }^{1}$ B Tümmler ${ }^{7}$
}

${ }^{1}$ Department of Pediatrics, CF Center, University Hospital of Jena, Germany; ${ }^{2}$ Department of Pediatrics, CF Center, University of Erlangen-Nuernberg,

Germany; ${ }^{3}$ CF Center, Chemnitz Hospital, Germany;

${ }^{4}$ Otorhinolaryngology, University of Erlangen-Nuernberg,

Germany; ${ }^{5}$ Otorhinolaryngology, University Hospital of Jena, Germany; ${ }^{6}$ Department of

Medical Informatics and Biometrics, University of Dresden, Germany; ${ }^{7}$ Klinische Forschergruppe, Pediatric Pneumology, Medizinische Hochschule Hannover, Germany; ${ }^{8}$ Institute of Medical Microbiology, University Hospital of Jena, Germany; ${ }^{9}$ Institute of Medical Microbiology, University Hospital Münster, Germany

Correspondence to:

Dr J G Mainz, Cystic Fibrosis

Center, Department of

Pediatrics, University of Jena, Kochstrasse 2, D-07740 Jena, Germany; Jochen.Mainz@ med.uni-jena.de

Received 11 July 2008 Accepted 25 February 2009

Published Online First

11 March 2009

\section{ABSTRACT}

Rationale: Lower airway (LAW) infection with

Pseudomonas aeruginosa and Staphylococcus aureus is the leading cause of morbidity in cystic fibrosis (CF). The upper airways (UAW) were shown to be a gateway for acquisition of opportunistic bacteria and to act as a reservoir for them. Therefore, tools for UAW assessment within CF routine care require evaluation.

Objectives: The aims of the study were non-invasive assessment of UAW and LAW microbial colonisation, and genotyping of $P$ aeruginosa and $S$ aureus strains from both segments.

Methods: 182 patients with CF were evaluated lage 0.4-68 years, median 17 years). LAW specimens were preferably sampled as expectorated sputum and UAW specimens by nasal lavage. $P$ aeruginosa and $S$ aureus isolates were typed by informative single nucleotide polymorphisms (SNPs) or by spa typing, respectively. Results: Of the typable $S$ aureus and $P$ aeruginosa isolates from concomitant UAW- and LAW-positive specimens, 31 of 36 patients were carrying identical $S$ aureus spa types and 23 of 24 patients identical $P$ aeruginosa SNP genotypes in both compartments. Detection of $S$ aureus or $P$ aeruginosa in LAW specimens was associated with a 15- or 88-fold higher likelihood also to identify $S$ aureus or $P$ aeruginosa in a UAW specimen from the same patient.

Conclusions: The presence of identical genotypes in UAW and LAW suggests that the UAW play a role as a reservoir of $S$ aureus and $P$ aeruginosa in CF. Nasal lavage appears to be suitable for non-invasive UAW sampling, but further longitudinal analyses and comparison with invasive methods are required. While UAW bacterial colonisation is typically not assessed in regular CF care, the data challenge the need to discuss diagnostic and therapeutic standards for this airway compartment.

Trial registration number: NCT00266474.

Cystic fibrosis (CF) is the most frequent lifethreatening recessive genetic disorder in Caucasians. It is caused by mutations in the Cystic Fibrosis Transmembrane conductance Regulator (CFTR) gene. Chronic pulmonary infection with opportunistic bacteria is the major cause of morbidity and mortality in CF. Staphylococcus aureus is one of the first pathogens infecting CF airways for extended periods. ${ }^{12}$ Later, up to $80 \%$ of adults with CF are chronically colonised with Pseudomonas aeruginos $a,{ }^{3}$ indicating progression of the pulmonary destruction ${ }^{4}$ although aggressive antibiotic treatment can improve life expectancy. ${ }^{5}$ The natural course of the disease can be ameliorated by preventing or postponing chronic $P$ aeruginosa colonisation. $P$ aeruginosa eradication is possible in early colonisation as long as colonies do not evolve to mucoid phenotypes. Therefore, further understanding of pathways leading to $P$ aeruginosa acquisition is a key issue of contemporary CF research.

Similar to the lower airways (LAW), mucociliary clearance of the upper airways (UAW) is impaired by the causative CFTR defect. Therefore, mucus retention, chronic inflammation, and colonisation of the UAW with opportunistic bacteria are typical in CF. Chronic rhinosinusitis and nasal polyps are pathognomonic signs of the disease. ${ }^{6-8}$ Resulting symptoms are chronic nasal congestion, rhinorrhoea with anterior or postnasal drip, mouthbreathing, facial pain, anosmia and sleep disturbances, which impair overall health. Altogether, $30-67 \%$ of the patients are reported to suffer from chronic rhinosinusitis over all age groups. ${ }^{78}$ Morphological abnormalities of the paranasal sinuses are detected by CT in almost $100 \%$ of patients, ${ }^{9}$ although in some cases this is due to hypoplasic frontal sinuses.

Sinonasal involvement in CF has been proposed as the major source for chronic bronchopulmonary infection with opportunistic bacteria. ${ }^{7} S$ aureus and $P$ aeruginosa are known to colonise the UAW in those with $\mathrm{CF}^{10}$ and therefore this site may function as a gateway and reservoir for subsequent pulmonary infection. If this were generally true in CF, the same bacterial clones should inhabit the UAW and LAW, and the detection of early airway colonisation in UAW and subsequent therapeutic intervention could accordingly improve the success rate of effective eradication. ${ }^{11}$ However, despite its potential impact on the management of CF, the association between UAW and LAW colonisation has never been systematically investigated. Hence, we set up a prospective clinical study to compare the microbial flora at the same time in upper and lower CF airways and to assess the genetic relatedness between $S$ aureus and $P$ aeruginosa strains inhabiting the UAW and LAW of subjects with CF. Moreover, non-invasive and simple methods for sampling the UAW that are applicable to daily clinical practice were compared in terms of their sensitivity.

\section{MATERIALS AND METHODS}

\section{Patients}

A total of 187 patients from five German CF outpatient clinics were enrolled in the study between December 2005 and April 2007. Microbiological sampling from both UAW and LAW was performed for 182 patients. 
The inclusion criterion was an established diagnosis of CF confirmed by at least three positive sweat tests and/or two disease-causing CFTR mutations. Exclusion criteria were sinonasal surgery $\leqslant 6$ months prior to recruitment and systemic antimicrobial $P$ aeruginosa therapy. Treatment with inhalative aminoglycosides and oral macrolides was reported. Patients were excluded from nasal lavage if they suffered from recurrent nasal bleeding, if the tympanum was perforated and/or if cooperation was not possible (age or other reasons).

Informed written consent was obtained from patients and their parental guardians. The study was approved by the local ethics committees.

\section{Rhinoscopy}

Nasal examination performed by flexible or rigid endoscopy included assessment of the mucosal status, sizing of nasal polyps, if applicable, and evaluation of secretions, crusts and other pathological alterations.

\section{Nasal lavage}

Nasal lavage was performed by inserting $10 \mathrm{ml}$ of sterile isotonic saline into each nostril with a $10 \mathrm{ml}$ syringe with a slightly reclined position of the head during occlusion of the soft palate (fig 1), as performed during standard therapeutic nasal lavage. ${ }^{12}$ Prior to the collection of nasal lavage, visual aids and verbal instructions were provided. Samples with contamination from the oral cavity were discarded and the procedure was repeated until non-contaminated samples were retrieved.

In general, patients older than 6 years were able to perform nasal lavage accurately. Alternatively, deep nasal swabs or swabs of nasal secretions sneezed into a handkerchief (blowing samples) were taken. Additionally, sputum samples or deep throat swabs were obtained. Specimens were processed by the local microbiology laboratory according to the German quality assurance guidelines for CF microbiology. ${ }^{13}$

\section{Molecular typing of $\boldsymbol{P}$ aeruginosa and $\boldsymbol{S}$ aureus}

$P$ aeruginosa isolates were typed in 16 SNPs with a custom-made microarray as described previously. ${ }^{14}$ DNA amplified from the bacterial colony by cycles of multiplex primer extension was

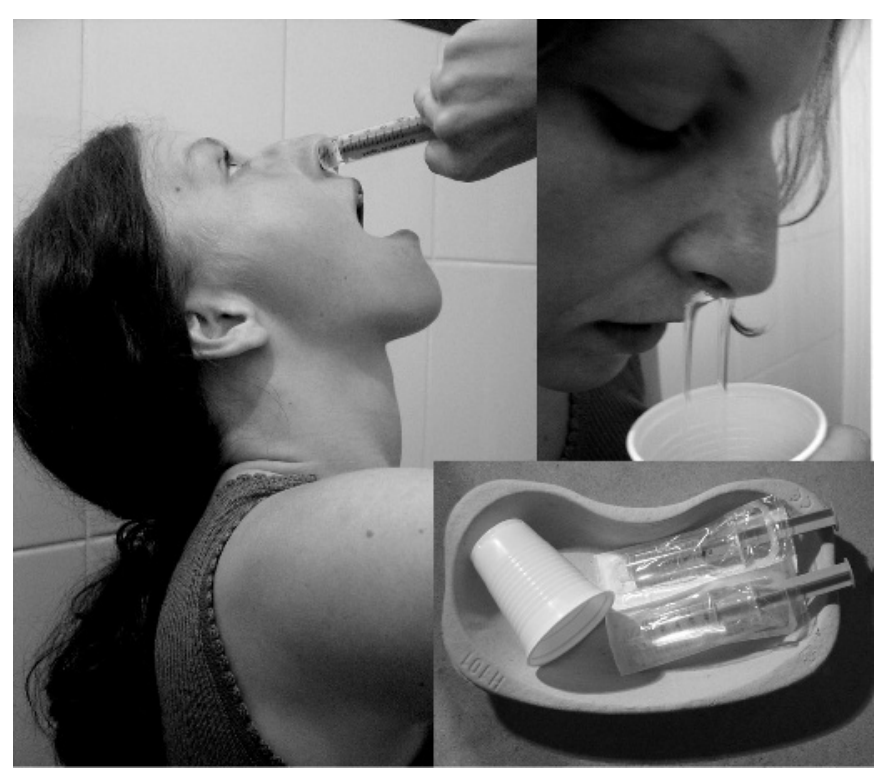

Figure 1 Method of diagnostic nasal lavage. hybridised onto the microarray to yield an electronically portable binary multimarker genotype. ${ }^{14} \mathrm{~S}$ aureus isolates were analysed by spa typing. Briefly, the variable region of protein A was amplified with the following primers: spa-1113f (5'-TAAAGACGATCCTTCGGTGAGC-3') and spa-1514r $\left(5^{\prime} \text {-CAGCAGTAGTGCCGTTTGCTT-3' }\right)^{15}$ and sequenced. Spa types were determined with the Ridom StaphType software. ${ }^{16}$ Numeric spa repeat and type codes were assigned.

\section{Statistics}

Data were evaluated with SPSS for Windows Version 11.5.1 (SPSS, Chicago, Illinois, USA) and Microsoft Excel 2003 (Microsoft, Redmond, Washington, USA). We performed explorative analyses of data. The association between detection of bacteria and fungi in the UAW and LAW was estimated by phi-analysis. Agreement or concordance between both compartments (beyond what would be expected by chance) was measured by Cohen's kappa. p Values of kappa below 0.05 (global alpha) indicate a statistically significant difference from only chance agreement. Bonferroni adjustment was applied to control alpha for multiple comparisons. To describe the relative risk of finding bacteria or fungi in a UAW sample, we used the odds of finding them in LAW-positive and in LAW-negative subjects and calculated odds ratios (ORs) and their 95\% CIs.

\section{RESULTS}

\section{Demographic data}

Baseline data from the 187 patients with CF (81 females/106 males) recruited in the five outpatient clinics are presented in table 1.

The mutation F508del was detected in 163/187 patients (87.7\%), with $78(41.7 \%)$ being homozygous for this most frequent CFTR defect in the German CF population. G551D was detected in 20 patients (5.3\%), N1303K and G542X in 10 patients each $(2.7 \%), \mathrm{R} 553 \mathrm{X}$ in 9 patients (2.4\%), R347P in 8 patients $(2.1 \%)$ and $3849+10 \mathrm{kbC}>\mathrm{T}$ in 7 patientss $(1.9 \%)$. Exogenous pancreatic insufficiency was present in 175/187 patients (93.6\%), and 25/187 (13.4\%) were diabetic. Altogether, allergic sensitisation was found in 89/145 (61.4\%) assessed patients by detection of specific immunoglobulin $\mathrm{E}$ ( $\mathrm{IgE}$ ) in serum. Of the 187 patients with CF, 36 (19.3\%) presented with allergic rhinitis and $14 / 187(7.5 \%)$ fulfilled the criteria for allergic bronchopulmonary aspergillosis (ABPA), according to the CF consensus guidelines. ${ }^{19}$ ENT (Ear, nose and throat) surgery had previously been performed in 79 of 187 recruited patients with CF, corresponding to a total percentage of $42.2 \%$.

Table 1 Age, pulmonary function, body weight-related parameters and Shwachman-Kulczycki score of the included patients

\begin{tabular}{lccc}
\hline & $\begin{array}{l}\text { Median (inner } \\
\text { quartiles) }\end{array}$ & Range & No \\
\hline Age (years) & $17(8-24)$ & $0.4-68$ & 187 \\
FEV $_{1}(\%$ predicted) & $83(60-102)$ & $17-150$ & 161 \\
FVC (\% predicted) & $87(72-96)$ & $32-121$ & 162 \\
FEF25 (\% predicted) & $48(19-73)$ & $6-170$ & 160 \\
FEF75/25 (\% predicted) & $62(25-90)$ & $4-163$ & 121 \\
RV (\% predicted) & $148(112-203)$ & $42-440$ & 152 \\
BMI ( $\geqslant 18$ years) (kg/m $\left.{ }^{2}\right)$ & $20(18-23)$ & $15-33$ & 84 \\
BMI $\left(<18\right.$ years) (percentile) ${ }^{17}$ & $35(21-56)$ & $1-93$ & 102 \\
Shwachman score (without RX) ${ }^{18}$ & $70(60-75)$ & $25-75$ & 187 \\
\hline
\end{tabular}

BMI, body mass index; FEF25, forced expiratory flow at $25 \%$ of the forced vital capacity; FEF75/25, forced expiratory flow at $75-25 \%$ of the forced vital capacity; $\mathrm{FEV}_{1}$, forced expiratory volume in $1 \mathrm{~s}$; FVC, forced vital capacity; RV, residual volume; $\mathrm{RX}$, chest roentgenogram. 
Table 2 Bacteria and fungi in the upper and lower airways from 182 individuals with cystic fibrosis

\begin{tabular}{|c|c|c|c|c|c|c|c|}
\hline & \multirow{2}{*}{$\begin{array}{l}\begin{array}{l}\text { Detection } \\
\text { in LAW }\end{array} \\
\text { No }\end{array}$} & \multirow{2}{*}{$\begin{array}{l}\begin{array}{l}\text { Detection } \\
\text { in UAW }\end{array} \\
\text { No }\end{array}$} & \multicolumn{3}{|c|}{$\begin{array}{l}\text { Detection in both } \\
\text { compartments }\end{array}$} & \multirow[b]{2}{*}{ Карра* } & \multirow[b]{2}{*}{ p Value } \\
\hline & & & No & $\begin{array}{l}\% \text { of } \\
\text { UAW }\end{array}$ & $\begin{array}{l}\% \text { of } \\
\text { LAW }\end{array}$ & & \\
\hline Pseudomonas aeruginosa (total) & 65 & 29 & 28 & 96.6 & 43.1 & 0.48 & $<0.001$ \\
\hline Pseudomonas aeruginosa (mucoid) & 45 & 21 & 18 & 85.7 & 40.0 & 0.46 & $<0.001$ \\
\hline Pseudomonas aeruginosa (non-mucoid) & 31 & 11 & 4 & 36.4 & 12.9 & 0.11 & 0.095 \\
\hline $\begin{array}{l}\text { Pseudomonas alcaligenes/Comamonas } \\
\text { testosteroni }\end{array}$ & 2 & 3 & 1 & 33.3 & 50.0 & 0.39 & 0.033 \\
\hline Pseudomonadaceae ssp. & 3 & 4 & 1 & 25.0 & 33.3 & 0.27 & 0.065 \\
\hline Staphylococcus aureus (without MRSA) & 60 & 52 & 38 & 73.1 & 63.3 & 0.54 & $<0.001$ \\
\hline MRSA & 4 & 3 & 3 & 100.0 & 75.0 & 0.85 & $<0.001$ \\
\hline Haemophilus influenzae & 18 & 11 & 6 & 54.5 & 33.3 & 0.37 & $<0.001$ \\
\hline Coagulase-negative staphylococci & 24 & 56 & 17 & 30.4 & 70.8 & 0.30 & $<0.001$ \\
\hline Streptococci ( $\alpha$-haemolytic) & 64 & 27 & 19 & 70.4 & 29.7 & 0.26 & $<0.001$ \\
\hline Streptococcus pneumoniae & 6 & 7 & 4 & 57.1 & 66.7 & 0.60 & $<0.001$ \\
\hline Streptococcus pyogenes ( $\beta$-haemolytic A) & 2 & 2 & 1 & 50.0 & 50.0 & 0.49 & 0.022 \\
\hline$\gamma$-Haemolytic streptococci & 20 & 9 & 5 & 55.6 & 25.0 & 0.30 & 0.001 \\
\hline Enterococci & 12 & 8 & 1 & 12.5 & 8.3 & 0.05 & 1 \\
\hline $\begin{array}{l}\text { Micrococcus, Lactococcus, Stomatococcus, } \\
\text { Sarcina }\end{array}$ & 5 & 1 & 0 & 0.0 & 0.0 & 0.00 & 1 \\
\hline Corynebacteria & 2 & 13 & 1 & 7.7 & 50.0 & 0.12 & 0.138 \\
\hline Neisseria ssp. & 26 & 11 & 5 & 45.5 & 19.2 & 0.20 & 0.01 \\
\hline Moraxella & 1 & 7 & 0 & 0 & 0 & 0.00 & 1 \\
\hline Acinetobacter baumannii & 1 & 1 & 0 & 0 & 0 & 0.00 & 1 \\
\hline Ralstonia pickettii & 4 & 2 & 2 & 100.0 & 50.0 & 0.66 & $<0.001$ \\
\hline Stenotrophomonas maltophilia & 18 & 2 & 2 & 100.0 & 11.1 & 0.18 & 0.009 \\
\hline Ochrobactrum anthropi & 2 & 2 & 1 & 50.0 & 50.0 & 0.49 & 0.022 \\
\hline Escherichia coli & 4 & 1 & 1 & 100.0 & 25.0 & 0.40 & 0.022 \\
\hline Enterobacter cloacae & 4 & 1 & 0 & 0 & 0 & 0.00 & 1 \\
\hline Klebsiella oxytoca, $K$ terrigena, $K$ planticola & 4 & 2 & 1 & 50.0 & 25.0 & 0.32 & 0.044 \\
\hline Proteus mirabilis & 2 & 2 & 2 & 100.0 & 100.0 & 1.00 & $<0.001$ \\
\hline Serratia marcescens, $S$ proteamaculans & 2 & 2 & 0 & 0 & 0 & 0.00 & 1 \\
\hline $\begin{array}{l}\text { Morganella, Providencia, Edwardsiella, } \\
\text { Hafnia }\end{array}$ & 1 & 2 & 0 & 0 & 0 & 0.00 & 1 \\
\hline Haemophilus parainfluenzae & 12 & 3 & 1 & 33.3 & 8.3 & 0.11 & 0.186 \\
\hline Candida spp. & 31 & 4 & 1 & 25.0 & 3.2 & 0.02 & 1 \\
\hline Candida albicans & 33 & 0 & 0 & 0 & 0 & & \\
\hline Aspergillus fumigatus & 26 & 1 & 1 & 100.0 & 3.8 & 0.06 & 0.143 \\
\hline Penicillium & 2 & 1 & 0 & 0 & 0 & 0.00 & 1 \\
\hline
\end{tabular}

${ }^{*} A$ kappa value of 0 indicates only chance agreement, and a value of 1 indicates complete agreement.

†Local $p$ values are given; after Bonferroni procedure only local $p$ values $<0.0015$ are statistically significant for the global alpha of 0.05 .

LAW, lower airways; MRSA, methicillin-resistant Staphylococcus aureus; UAW, upper airways.

\section{Upper and lower airway colonisation}

A total of 182 patients were assessed for microbial colonisation in LAW and UAW (see table 2).

Haemophilus influenzae was found in 9.9\% (18) of LAW and $6.0 \%$ (11) of UAW samples, and in 6 of these, $H$ influenzae was detected in both compartments. Aspergillus fumigatus was recovered in $14.3 \%(26 / 182)$ of LAW cultures, whereas it grew in only one UAW sample that had been retrieved from a patient who simultaneously had a positive LAW culture. Candida albicans was found in $18.1 \%$ (33) of the LAW cultures but in none of the UAW samples.

\section{$P$ aeruginosa}

History of chronic colonisation of the airways with $P$ aeruginosa was defined according to Kerem et $a^{20}$ as having at least $50 \%$ of $P$ aeruginosa-positive LAW samples during a period of 12 months. A total of 63 of 182 (34.6\%) patients fulfilled the criteria of history of chronic colonisation with $P$ aeruginosa

$P$ aeruginosa could be identified in UAW samples from 29 (46.0\%) of the 63 patients with a history of chronic $P$ aeruginosa colonisation of the LAW. At the same time, the bacterium was found in $90.5 \%(57 / 63)$ of the LAW cultures from these patients. The proportion of patients with detection of $P$ aeruginosa in both airway segments increased with age ${ }^{13}$ (fig 2).

In one patient with a history of chronic pulmonary colonisation, $P$ aeruginosa could only be detected in the nasal lavage but not in sputum. $P$ aeruginosa was 88-fold more likely to be detectable in a UAW sample from a LAW $P$ aeruginosapositive subject than from a LAW $P$ aeruginosa-negative subject (95\% CI 11.5 to 667.5$)$.

\section{$S$ aureus}

$S$ aureus was recovered in 55 UAW (30\%) and 64 (35\%) LAW specimens. $S$ aureus isolates of 4/64 LAW specimens were methicillin-resistant $S$ aureus (MRSA). Samples from 41/64 (64\%) patients were positive for $S$ aureus in both the UAW and LAW specimens. Samples from 14 patients were only positive for $S$ aureus in the UAW specimen, while samples from 23 patients were only positive in LAW specimens. $S$ aureus colonisation of both airway sites was found in all age groups 


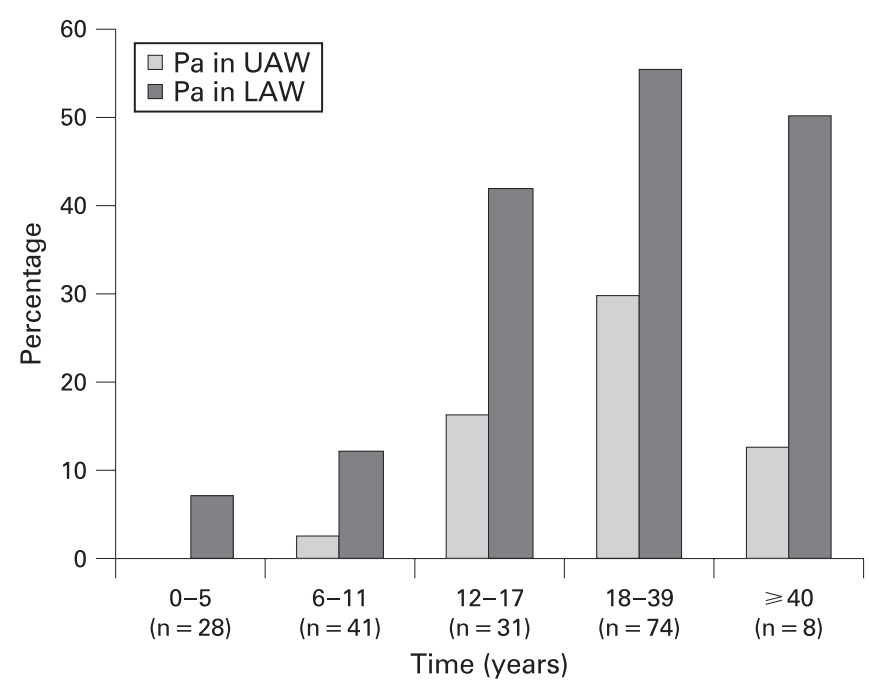

Figure 2 Fraction of patients with detection of Pseudomonas aeruginosa $(\mathrm{Pa})$ in the upper (UAW) and lower airways (LAW) in relation to their age $(n=182)$.

(fig 3). A statistically significant association for $S$ aureus carriage in both airway sites was found $(\Phi=0.568, \mathrm{p}<0.001)$. The OR analysis revealed a LAW $S$ aureus carrier to be 15.4-fold more likely to be $S$ aureus positive in the UAW than a LAW $S$ aureusnegative subject (95\% CI 7.1 to 33.4 ).

Patients with a history of chronic $P$ aeruginosa colonisation less frequently carried $S$ aureus in the UAW and LAW. $S$ aureus was found in the UAW of $14.3 \%(9 / 63)$ of the patients with chronic $P$ aeruginosa colonisation of the LAW, but in $38.7 \%$ $(46 / 119)$ of the patients without chronic $P$ aeruginosa colonisation. Accordingly, the OR was 3.8 higher for nasal $S$ aureus detection in $P$ aeruginosa-negative patients (95\% CI 1.7 and 8.4 ).

\section{Genetic relatedness of $\boldsymbol{P}$ aeruginosa and $\boldsymbol{S}$ aureus strains detected in the UAW and LAW}

$P$ aeruginosa strains of 29 patients were typed in 16 informative SNPs of the core genome. ${ }^{14}$ Isolates from both UAW and LAW were available from 24 patients (LAW samples, 21 expectorated sputum and 3 deep throat swabs; UAW, all 24 obtained by nasal

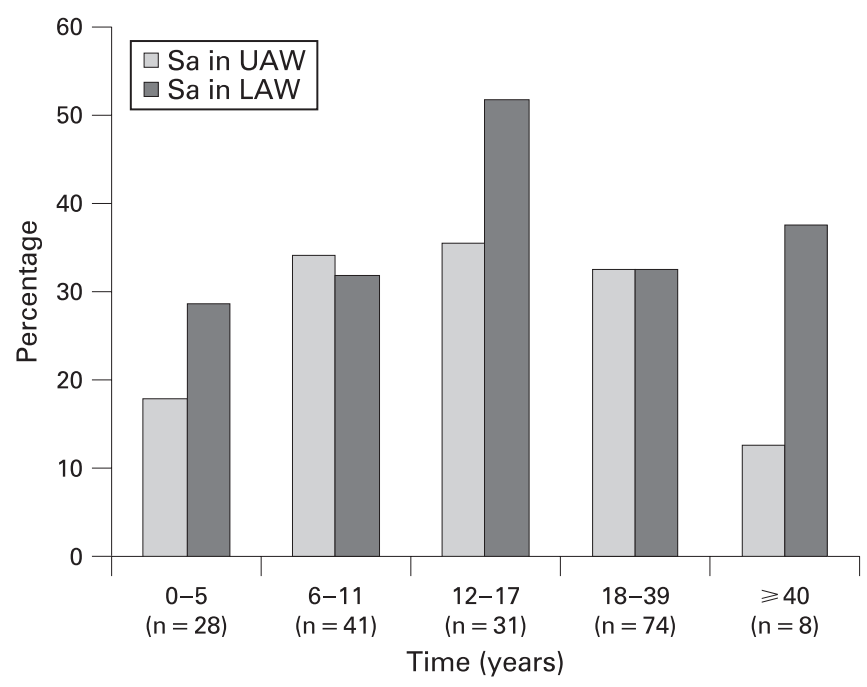

Figure 3 Patients with Stapylococcus aureus (Sa) in the upper (UAW) and lower airways (UAW) in relation to age $(n=182)$. lavage). Strain pairs with identical $P$ aeruginosa SNP marker genotype had been retrieved from the UAW and LAW specimens of 23 patients. Distinct $P$ aeruginosa genotypes had been recovered from the UAW and LAW of only one patient (fig 4A). The isolates were classified into 21 different genotypes including eight common genotypes that each make up $>1 \%$ of the $P$ aeruginos a population. ${ }^{14}$ Seven genotypes of this study had not been observed before in our collection of $>2000$ strains that represent 180 independent clones.

$S$ aureus isolates from 36 patients were analysed further. The isolates were cultured from nasal swabs $(n=30)$ or nasal lavage $(\mathrm{n}=14)$ representative for the UAW, or from deep throat swabs $(n=24)$ or sputum $(n=12)$ representative for the LAW. All $S$ aureus isolates were subjected to spa typing ${ }^{15}$ that allows comparison of the $S$ aureus isolates not only within the same patient but also between different patients and between different centres. In most patients, the isolates from the UAW and LAW were genotypically identical (31/36; 86.1\%) (fig 4B). More than one $S$ aureus genotype was retrieved from throat swabs of two patients. The isolates of 36 patients revealed 37 different spa types. Only two of three MRSA couplets from both airway segments were available for typing. They were found to be genotypically identical in the UAW and LAW for each patient.

Assessment of different methods to sample the UAW and LAW Comparison of different methods to sample material from the UAW of 61 patients revealed remarkable discrepancies in the rate of $P$ aeruginosa-positive cultures from patients with a history of chronic pulmonary $P$ aeruginosa colonisation. $P$ aeruginosa was detected in $56 \%$ of nasal lavages (25/45, $\Phi=0.648 . \quad p<0.001), 29 \%$ of deep nasal swabs $(11 / 38$, $\Phi=0.469, \quad p<0.001)$ and $10 \%$ of blowing samples $(1 / 10$, $\Phi=0.182, p=0.667)$. In the case of the LAW specimens, $P$ aeruginosa was detected in 51 of 56 sputa and 6 of 7 deep throat swabs (fig 5).

Of the 53 patients whose UAW were sampled simultaneously with nasal lavage and nasal swab, 7 patients were $P$ aeruginosa positive with both methods, 4 patients $P$ aeruginosa were positive only with lavage, and one patient only with nasal swab.

\section{DISCUSSION}

Chronic rhinosinusitis is a hallmark of $\mathrm{CF}^{6-9}$ The local symptoms frequently impair the quality of life, but-more importantly - chronic rhinosinusitis may remodel the UAW to become a niche for opportunistic pathogens that may descend into the lungs during periods of damage of the oropharyngeal barrier.

This study simultaneously sampled the UAW and LAW of patients with CF with non-invasive methods. The cultured bacteria and fungi were classified into 30 groups (table 2). In $50 \%$ of cases, the same microorganism was also recovered from the lungs if it had been cultured from the UAW. A few pathogens were predominantly recovered from only one habitat. Coagulase-negative staphylococci, Corynebacteria and Moraxella spp. were frequently cultured from the UAW, but infrequently from the lungs, in accordance with their preponderance in the nasopharyngeal tract as their natural niche in healthy carriers. ${ }^{21}$ On the other hand, opportunistic pathogens ${ }^{21}$ that typically are absent in the commensal flora of healthy individuals were more frequently cultured from CF LAW than from CF UAW. Examples are Candida and Aspergillus, 
A

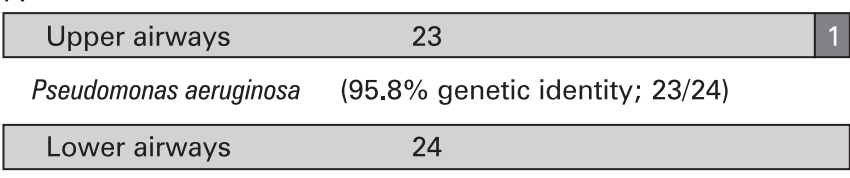

$\square$ Identical genotype

$\square$ Dissimilar genotype

B

\begin{tabular}{|cc|c|}
\hline Upper airways & 31 & 6 \\
\hline Staphylococcus aureus & $(83.8 \%$ genetic identity; 31/37) \\
\hline Lower airways & 37 \\
\hline
\end{tabular}

Figure 4 Genetic relatedness of (A) Pseudomonas aeruginosa and (B) Staphylococcus aureus isolates from the upper and lower airways of individuals with cystic fibrosis.

$H$ influenzae and $H$ parainfluenzae, Stenotrophomonas maltophilia and the leading pathogen in CF, $P$ aeruginosa (table 2).

Mucus retention and insufficient aeration of the paranasal sinuses, and nasal polyps and congestion are obligatory symptoms in CF. Hence, one may wonder why the reasonable and fundamental question of whether the UAW are a reservoir and gateway for microbial colonisation in CF has so far been addressed in only a few studies. ${ }^{22-24}$ The anatomical barriers to retrieve samples are one obvious reason. The direct assessment of the paranasal sinuses requires quite invasive methods such as endoscopy-guided sinus aspiration, ${ }^{25}$ sinus puncture or intraoperative sampling, ${ }^{22}$ which are unsuitable for routine clinical management. Thus, this study evaluated an alternative option of non-invasive methods to sample the UAW for bacteriological analysis. The detection of $P$ aeruginosa in UAW specimens retrieved from patients with chronic airway colonisation with $P$ aeruginosa was taken as the standard for comparison. Recovery of $P$ aeruginosa from LAW specimens (sputum or deep throat swab) was about $90 \%$. Nasal lavage turned out to be the method of choice for sampling the UAW, with a recovery rate of close to $60 \%$ of $P$ aeruginosa-positive cultures. If subjects were unable to perform the manoeuvre, blowing samples or deep nasal swabs were taken. However, these alternative procedures

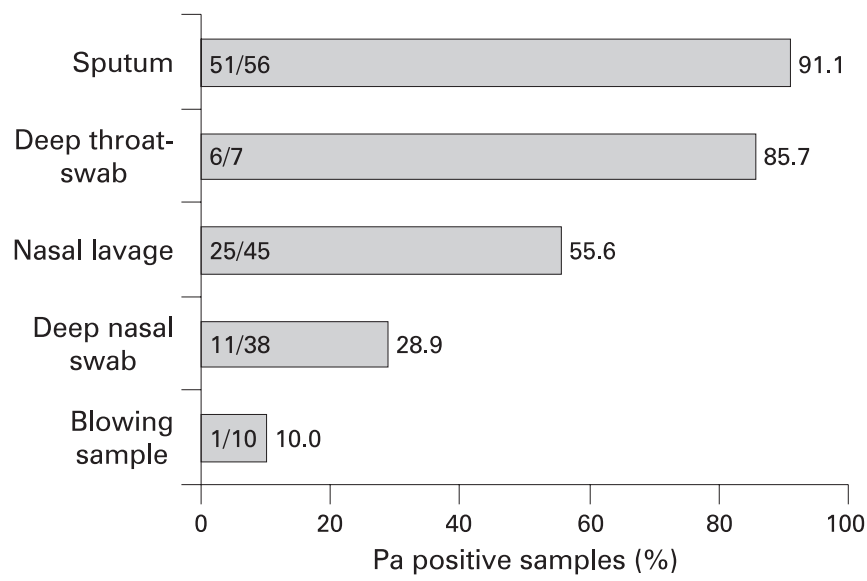

Figure 5 Recovery of $P$ aeruginosa $(\mathrm{Pa})$ in specimens taken from the upper (UAW) or lower airways (LAW) of patients with a history of chronic $P$ aeruginosa colonisation of the lower airways (UAW, $n=183$; LAW, $\mathrm{n}=184$ ). were not sensitive enough and therefore have to be classified as inappropriate.

The sensitivity of nasal lavage can probably be improved with the subject's increasing experience in sampling. Although we provided meticulous visual and oral instructions to each study subject, some patients did not adhere to the protocol. Instead of rinsing the full volume of $10 \mathrm{ml}$ of saline, they just applied portions of 2-3 $\mathrm{ml}$ with their head in the forward position. Supposedly these individuals predominantly rinsed the nasal vestibulum, but not the nasal conchae with the orifices of the paranasal sinuses. In conclusion, nasal lavage seems to be a promising technique for sampling the UAW in clinical practice. The subject needs to be trained as is necessary for lung function testing. Since both methods require the subject's cooperation, they are not suitable for young children. However, it is exactly this group of young minors who would benefit most from an early detection of microbial pathogens in the UAW prior to their descent into the LAW. In the case of the major pathogen $P$ aeruginosa, numerous studies have convincingly demonstrated a $80 \%$ success rate of eradication of $P$ aeruginosa from the airways $^{526}$ during the stage of early colonisation prior to bacterial adaptation to and diversification in the niche. ${ }^{27} 28$ Considering the impact of the prevention and therapy of infection, the protocols of UAW sampling for bacteriological analysis, particularly for the risk group of young children, should be further optimised in prospective trials and then introduced into the management of CF.

$S$ aureus and $P$ aeruginosa are the dominant pathogens in CF. ${ }^{1{ }^{2}{ }^{28}}$ Therefore, the major goal of our multicentre study was the analysis of the genetic relatedness of $S$ aureus and $P$ aeruginosa strains that had been retrieved at the same time from the UAW and LAW of a subject with CF. To address this issue, the currently most informative molecular typing methods for $S$ aureus $^{15}$ and $P$ aeruginosa ${ }^{14}$ were applied. The outcome was unequivocal; $86 \%$ of the $S$ aureus strain pairs and $95 \%$ of the $P$ aeruginosa strain pairs were genotypically identical. In other words, the majority of $S$ aureus carriers and almost all $P$ aeruginosa carriers are harbouring the same clones in the upper airways and the lungs. Therefore, the UAW and LAW of a patient with CF are colonised with the same populations of $S$ aureus and $P$ aeruginosa.

This major finding based on the prospective analysis of a CF cohort of all age groups and grades of disease severity is supported by the few published data on preselected patient subgroups. ${ }^{22-24}$ Walter et al ${ }^{24}$ typed LAW $P$ aeruginosa isolates from patients prior to and after lung transplantation. Within a few months, the $P$ aeruginos $a$-free donor lungs became colonised with the $P$ aeruginosa genotype of the explanted lungs. The authors concluded that the patients' UAW were the reservoir for the colonisation of the lung transplant. During end-stage CF pulmonary disease, the UAW and LAW were colonised with the same $P$ aeruginosa clones. This conclusion was found to be true also for less advanced stages of lung disease. Taylor et $a^{23}$ identified the same $P$ aeruginosa macrorestriction fragment genotypes in sputa and UAW samples from adults with CF. Recently, Muhlebach et al2 compared the bacteriology of the sinus with that of oropharyngeal swabs and bronchoalveolar lavage fluid (BALF) in children with CF. Samples were taken during operations on subjects requiring sinonasal surgery. If $S$ aureus or $P$ aeruginosa were isolated from both the sinus and the LAW specimen, the concordance of genotypes was $83 \%$. This figure corresponds reasonably well with our data on LAW and UAW strain pairs retrieved by non-invasive procedures. 
Considering the possible role of the UAW as a reservoir for lung infections with the major CF pathogens that has been demonstrated by both our study and that of Muhlebach et al, future comparative investigations on the recovery of culturable microbes by invasive and non-invasive sampling of the UAW will be of great interest. If the current protocol of nasal lavage or a modified version would achieve similar detection rates to the direct analysis of sinonasal tissue, nasal lavage could become a routine measure for the bacteriological surveillance in CF and a valid surrogate parameter for antimicrobial intervention trials. Lastly, our results disprove earlier claims ${ }^{29}{ }^{30}$ that the sinonasal compartment is not a reservoir for the descending infection to the lung in CF. On the contrary, as several authors had postulated previously, ${ }^{23}{ }^{31-33}$ the UAW appear to play a role in the acquisition and persistence of opportunistic bacteria in CF which requires further investigation. ${ }^{34}$

Acknowledgements: We thank Dr Guenther Frey and Dr Karin Thoss for enrolling patients from their outpatient clinics, and Christiane Ritschel from the Jena University CF study group for the substantial organisational help.

Funding: The study was supported by funds from Hoffmann-La Roche, Novartis, InfectoPharm, Pari and Gruenenthal. We confirm that the financial support did not influence any aspect of the study and that no other financial resources were provided.

Competing interests: None.

Ethics approval: The trial was approved by the Jena University Ethics Committee. Patient consent: Obtained.

Contributors: JM was principal investigator. LN and MK conducted and supervised the study in participating CF centres. SM and GS were coinvestigators in participating ENT units. JM contributed to the concept and design of the study together with BT, JFB, IS and MS. BT, BCK, LW, NC and WP performed the microbiological analyses including genotyping of $P$ aeruginosa and $S$ aureus strains. BT, BCK, IS, MS and BW contributed to the analysis and interpretation of data.

\section{REFERENCES}

1. Cystic Fibrosis Foundation. Patient Registry 2004. Annual Data Report. Bethesda, Maryland: Cystic Fibrosis Foundation, 2005.

2. Kahl BC, Duebbers A, Lubritz G, et al. Population dynamics of persistent Staphylococcus aureus isolated from the airways of cystic fibrosis patients during a 6-year prospective study. J Clin Microbiol 2003;41:4424-27.

3. Cystic Fibrosis Foundation Patient Registry. 2001 Annual Data Report to the Center Directors. Bethesda, MD: Cystic Fibrosis Foundation, 2002.

4. Henry RL, Mellis CM, Petrovic L. Mucoid Pseudomonas aeruginosa is a marker of poor survival in cystic fibrosis. Pediatr Pulmonol 1992;12:158-61.

5. Frederiksen B, Lanng $S$, Koch $\mathbf{C}$, et al. Improved survival in the Danish centertreated cystic fibrosis patients: results of aggressive treatment. Pediatr Pulmonol 1996;21:153-8.

6. Ramsey B, Richardson MA. Impact of sinusitis in cystic fibrosis. J Allergy Clin Immunol 1992:90:547-52.

7. Gysin C, Alothman GA, Papsin BC. Sinonasal disease in cystic fibrosis: clinical characteristics, diagnosis, and management. Pediatr Pulmonol 2000;30:481-9.

8. Coste A, Gilain L, Roger G, et al. Endoscopic and CT-scan evaluation of rhinosinusitis in cystic fibrosis. Rhinology 1995;33:152-156.

9. Eggesbo HB, Sovik S, Dolvik S, et al. CT characterization of developmental variations of the paranasal sinuses in cystic fibrosis. Acta Radiol 2001:42:482-93.
10. Shapiro ED, Milmoe GJ, Wald ER, et al. Bacteriology of the maxillary sinuses in patients with cystic fibrosis. J Infect Dis 1982;146:589-93.

11. Ratjen F. Changes in strategies for optimal antibacterial therapy in cystic fibrosis. Int J Antimicrob Agents 2001;17:93-6.

12. Pynnonen MA, Mukerji SS, Kim HM, et al. Nasal saline for chronic sinonasal symptoms: a randomized controlled trial. Arch Otolaryngol Head Neck Surg 2007;133:1115-20.

13. Hogardt M, Häußler S, Balke B, et al. Atemwegsinfektionen bei Mukoviszidose. Mikrobiologisch-infektiologische Qualitätsstandards (MiO) 24. München, Urban and Fischer, 2006

14. Wiehlmann L, Wagner G, Cramer N, et al. Population structure of Pseudomonas aeruginosa. Proc Natl Acad Sci USA 2007;104:8101-6.

15. Kahl BC, Mellmann A, Deiwick $S$, et al. Variation of the polymorphic region $X$ of the protein $\mathrm{A}$ gene during persistent airway infection of cystic fibrosis patients reflects two independent mechanisms of genetic change in Staphylococcus aureus. J Clin Microbiol 2005;43:502-5.

16. Harmsen D, Claus H, Witte W, et al. Typing of methicillin-resistant Staphylococcus aureus in the University hospital setting using a novel software for spa-repeat determination and database management. J Clin Microbiol 2003;41:5442-8.

17. Kromeyer-Hauschild K, Wabitsch M, Kunze D, et al. Perzentile für den Body-MassIndex für das Kindes- und Jugendalter unter Heranziehung verschiedener deutscher Stichproben. Monatsschr Kinderheilkd 2001;149:807-18.

18. Shwachman H, Kulczycki LL. Long-term study of one hundred five patients with cystic fibrosis; studies made over a five- to fourteen-year period. AMA J Dis Child 1958;96:6-15.

19. Stevens DA, Moss RB, Kurup VP, et al. Allergic bronchopulmonary aspergillosis in cystic fibrosis - state of the art: Cystic Fibrosis Foundation Consensus Conference. Clin Infect Dis 2003;37:225-264.

20. Kerem E, Corey M, Gold R, et al. Pulmonary function and clinical course in patients with cystic fibrosis after pulmonary colonisation with Pseudomonas aeruginosa. J Pediatr 1990;116:714-719.

21. Armstrong D, Cohen J. Infectious diseases. London, UK: Harcourt Publishers, 1999

22. Muhlebach MS, Miller MB, Moore C, et al. Are lower airway or throat cultures predictive of sinus bacteriology in cystic fibrosis? Pediatric Pulmonology 2006;:41:445-51.

23. Taylor RF, Morgan DW, Nicholson PS, et al. Extrapulmonary sites of Pseudomonas aeruginosa in adults with cystic fibrosis. Thorax 1992;47:426-8.

24. Walter S, Gudowius P, Bosshammer J, et al. Epidemiology of chronic Pseudomonas aeruginosa infections in the airways of lung transplant recipients with cystic fibrosis. Thorax 1997:52:318-21.

25. Cimmino M, Cavaliere M, Nardone $\mathbf{M}$, et al. Clinical characteristics and genotype analysis of patients with cystic fibrosis and nasal polyposis. Clin Otolaryngol Allied Sci 2003:28:125-32.

26. Wiesemann HG, Steinkamp G, Ratjen F, et al. Placebo-controlled, double-blind, randomized study of aerosolized tobramycin for early treatment of Pseudomonas aeruginosa colonization in cystic fibrosis. Pediatr Pulmonol 1998;25:88-92.

27. Smith EE, Buckley DG, Wu Z, et al. Genetic adaptation by Pseudomonas aeruginosa to the airways of cystic fibrosis patients. Proc Natl Acad Sci USA 2006;103:8487-92.

28. Bush A, Alton EWFW, Davies JC, et al. Cystic fibrosis in the 21st century. Basel, Switzerland: Karger, 2006.

29. Tsang V, Pitt TL, Kaufmann ME, et al. Colonisation of lung allografts with Pseudomonas aeruginosa in heart-lung transplant recipients with cystic fibrosis. Thorax 1994;49:721-2.

30. Batsakis JG, El-Naggar AK. Cystic fibrosis and the sinonasal tract. Ann Otol Rhinol Laryngol 1996;105:329-30.

31. Davidson TM, Murphy C, Mitchell M, et al. Management of chronic sinusitis in cystic fibrosis. Laryngoscope 1995;105:354-8.

32. Lewiston N, King V, Umetsu D, et al. Cystic fibrosis patients who have undergone heart-lung transplantation benefit from maxillary sinus antrostomy and repeated sinus lavage. Transplant Proc 1991;23:1207-8.

33. Nunley DR, Grgurich W, lacono AT, et al. Allograft colonization and infections with pseudomonas in cystic fibrosis lung transplant recipients. Chest 1998;113:1235-43.

34. Mainz JG, Koitschev A. Management of chronic rhinosinusitis in CF. J Cystic Fibrosis 2009:8S:10-4. 